\title{
Do-It-Yourself (DIY) based Flexible Paper Sensor Based Electronic System for Pill Health Monitoring
}

\author{
Sherjeel M. Khan \\ MMH Labs \\ King Abdullah University of Science and \\ Technology (KAUST) \\ Thuwal, Saudi Arabia \\ sherjeel.khan@kaust.edu.sa
}

\author{
Nadeem Qaiser \\ MMH Labs \\ King Abdullah University of Science and \\ Technology (KAUST) \\ Thuwal, Saudi Arabia \\ nadeem.qaiser@kaust.edu.sa
}

\author{
Muhammad M. Hussain \\ MMH Labs \\ King Abdullah University of Science and \\ Technology (KAUST) \\ Thuwal, Saudi Arabia \\ muhammadmustafa.hussain@kaust.edu
}

\begin{abstract}
Here we show, Do-It-Yourself (DIY) integration strategy of a low-cost paper sensor based flexible electronic system, using anisotropic conductive tape. The whole assembly is housed inside the cap of a prescription drug container to continuously monitor health of pills. These flexible modular decal sensors are connected with a central flexible decal electronic interface. Additionally, this simple easy-to-use DIY based integration strategy gives the flexibility to develop customized versions of the system depending on the requirements of different applications. This add-on system is easy to install or remove allowing easier implementation for practical use to monitor pill health using Internet-of-Things devices.
\end{abstract}

Keywords—pill health monitor, anisotropic conductive tape, DIY integration, paper sensor, flexible sensor

\section{INTRODUCTION}

Medicinal pill health and potency is affected by the storage conditions with ttemperature and humidity known to be the most important factors. High levels of temperatures and humidity exacerbate the degradation of pills [1]. Doctors recommend storage of most medicines at room temperature. For example, chitosan containing tablets should remain under $25{ }^{\circ} \mathrm{C}$ and $60 \% \mathrm{RH}$ (Relative Humidity) to maintain its potency [2]. Increased exposure to humid conditions beyond acceptable level change the appearance, hardness, mass and crystallinity of the medicine[3]. Nassar et al. have previously demonstrated the performance characteristics of paper-based humidity and temperature sensors for wearable applications. Those sensors showed consistent performance under various bending conditions [4]. Thus, we used the paper-based sensors with the purpose of monitoring the ambient environment inside the container. Generally, paper sensors are attached to respective electronic interface using conductive epoxy or soldering. This creates a redundancy in the proliferance of paper sensors as a low cost DIY based solution.

Here we show, using low-cost paper and stretchable sensors, we adopt a DIY integration strategy using anisotropic conductive tape to develop an affordable self-contained pill monitoring device can monitor pill health in real time. Since the functionality is kept modular, paper-based temperature and humidity sensors can be attached as needed to monitor the ambient environment inside the prescription container. This allows for an immediate on-demand response that can potentially save the patient from imminent death.

\section{DIY BASED SENSOR INTEGRATION AND ASSEMBLY}

\section{A. Containcer Ambience Monitoring Sensor Assembly}

We devised a simple method to convert paper sensors into modular decal sensors that can be attached to our electronic interface in any customized fashion. The paper sensors were converted into modular form by attaching conductive copper foil to the electrodes on the paper sensors. The sensors were then attached to a base structure (here onwards called a "sticker"). The sticker was made using Polyimide sheet (DuPont ${ }^{\mathrm{TM}}$ Kapton ${ }^{\circledR}$ 200HN Polyimide Film, 50 Micron Thickness) with pattern of interconnects formed using conductive copper tape as per the dimensions of the previously made modular paper sensors. Z-axis conductive tape (3M $\mathrm{M}^{\mathrm{TM}} \mathrm{Z}$ Axis Conductive Tape 9703) was placed on the area where the paper sensors needed to be attached. This Z-axis conductive tape conducts current anisotropically when two conductors are adhered firmly on the top and bottom sides of it. The modular paper sensors were then attached on their respective sites to make an electrical contact using only pressure from a finger between the sticker and the sensor. Fig 1. shows the prepared sticker with paper sensors attached on it. The conductive tape has embedded gold granules, allowing anisotropic conduction of electric current. The sticker was then placed on a bent platform to check the mechanical integrity of the connections under bending conditions as shown in Fig. 2. After several cycles of placement and removal from the bent structure, the sticker consistently preserved the same contact resistance.

\section{B. Containcer Ambience Monitoring Sensor Assembly}

Since we are using DIY strategy to make the whole system, the central electronic interface has to be made according to the decal sensors we created in the previous sections. Courtesy of our unique integration approach, we can make any desired decal system for a specific application in modular and

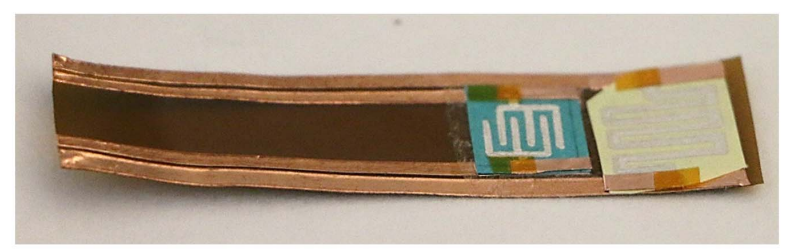

Fig. 1. Sticker with paper based humidity and temperature sensor attached using anisotropic consductive tape DIY integration appraoch 


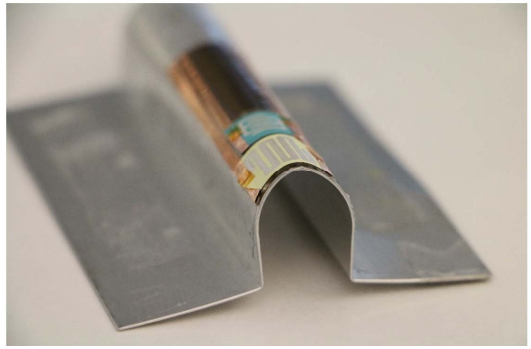

Fig. 3. Paper sensor sticker on a $1 \mathrm{~cm}$ radius bending platform

customizable fashion. Torres et al have successfully showed how bulk monocrystalline Silicon (100) based high performance Complementary Metal Oxide Semiconductor (CMOS) devices and circuitry can be flexed down to $0.5 \mathrm{~mm}$ bending radius [5]. We wanted to embed the system inside the prescription container, hence, we produced a fully flexible decal electronic interface. Figure 3 (left) shows a flexible microprocessor attached on a Polyimide sheet with connections made to the copper interconnects on the back side through polymer-vias and Figure 3 (right) shows modular paper sensor sticker attached to electronic interface sticker using the integration strategy discussed in sub-section A. The decal was placed on a $1 \mathrm{~cm}$ bending radius platform to demonstrate the overall flexibility of the whole system. This DIY based system can monitor temperature and humidity inside a drug container.

\section{Aniosotropic TAPE Electrical Contact Study}

In order to study the electrical behavior of the conductive tape, a $7 \mathrm{~mm}^{2}$ area of conductive tape was prepare on a slide and observed under a microscope. Fig. 4 (left) shows the image of conductive tape observed under a microscope where the silver particles responsible for conduction can be seen as black particles. Using "ImageJ" software we calculated the number of particles in a given area and their average size. In the observed sample we had an average of 360 articles with an average surface area of $2000 \mu \mathrm{m}^{2}$. We then recreated the model of the conductive tape in COMSOL and performed FEM simulations on the model. The model consisted of $7 \mathrm{~mm}^{2}$ of conductive tape with 360 silver particles equally distributed. The sample was covered on top and bottom with cooper electrodes provided with $5 \mathrm{~V}$ potential. Fig. 4 (right) shows the observed surface normal current density, It can be seen that the current density is concentrated within the areas lying above the silver particles. The current density is maximum around the area of contact with silver particles which proves the efficiency of electrical contact made using this integration technique. Fig. 5 shows potential drop across the top copper electrode, silver particles, and bottom copper electrode.
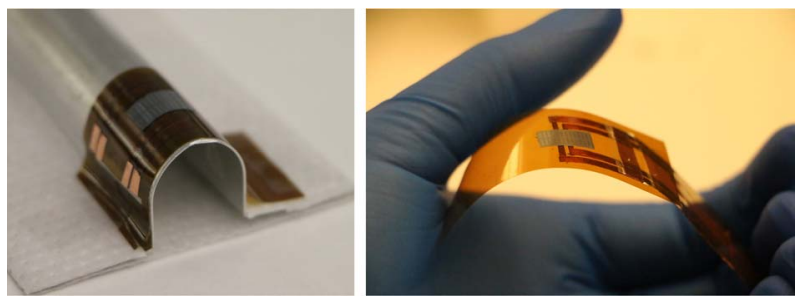

Fig. 5. Flexible microprocesse sticker on a $1 \mathrm{~cm}$ bending radius platform (left) and then connected to paper sensor sticker using conductive tape (right)

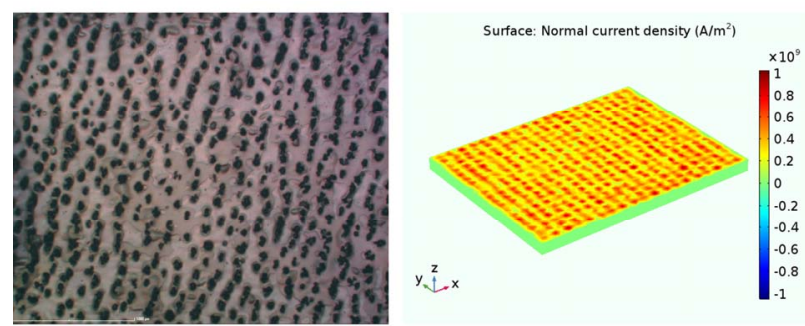

Fig. 2. $7 \mathrm{~mm}^{2}$ sample of anisotropic tape under microscope (left) and FEM simulation showing the observed current density for the sample (right)

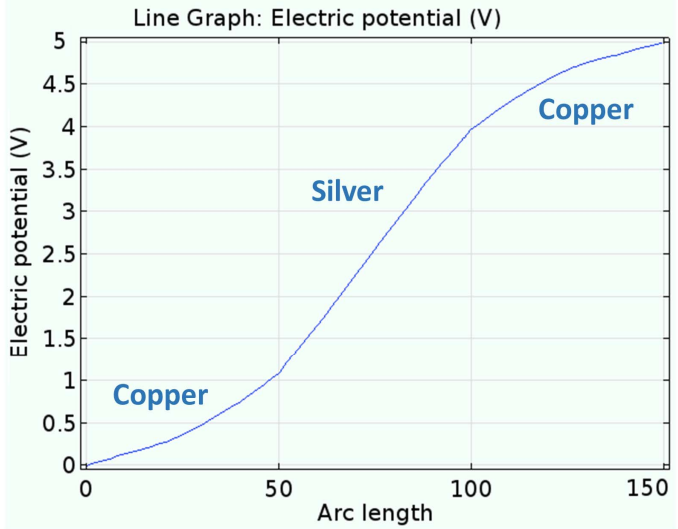

Fig. 4. Votlage potentail drop across copper-conductive tape-copper stack

\section{CONCLUSION}

We have previously demonstrated the performance of paper sensors as humidity and temperatures sensors. This new integration approach further facilities in the adoption of paper based sensors flexible system for low cost DIY based applications. The fabricated paper sensors and electronic interface can be placed inside a prescription bottle with minimal obtrusion to monitor the ambience inside the bottle for evaluation of pill health.

\section{REFERENCES}

[1] G. Pavlovska and S. Tanevska, "Influence of temperature and humidity on the degradation process of ascorbic acid in vitamin $\mathrm{C}$ chewable tablets," Journal of thermal analysis and calorimetry, vol. 111, no. 3, pp. 1971-1977, 2013

[2] J. M. Viljoen, J. H. Steenekamp, A. F. Marais, and A. F. Kotzé, "Effect of moisture content, temperature and exposure time on the physical stability of chitosan powder and tablets," Drug development and industrial pharmacy, vol. 40, no. 6, pp. 730-742, 2014.

[3] Y. Takekuma, H. Ishizaka, M. Sumi, Y. Sato, and M. Sugawara, "Difference in the Dissolution Behaviors of Tablets Containing Polyvinylpolypyrrolidone (PVPP) Depending on Pharmaceutical Formulation After Storage Under High Temperature and Humid Conditions," Journal of Pharmacy \& Pharmaceutical Sciences, vol. 19, no. 4, pp. 511-519, 2016.

[4] J. M. Nassar, K. Mishra, K. Lau, A. A. Aguirre - Pablo, and M. M. Hussain, "Recyclable Nonfunctionalized Paper - Based Ultralow - Cost Wearable Health Monitoring System," Advanced Materials Technologies, vol. 2, no. 4, 2017.

[5] J. P. Rojas et al., "Transformational silicon electronics," ACS nano, vol. 8, no. 2, pp. 1468-1474, 2014. 